Relations industrielles

Industrial Relations

\title{
L'Analyse des conventions collectives de travail
} Commentaires

\section{Jean-Réal Cardin}

Volume 17, numéro 1, janvier 1962

URI : https://id.erudit.org/iderudit/1037353ar

DOI : https://doi.org/10.7202/1037353ar

Aller au sommaire du numéro

Éditeur(s)

Département des relations industrielles de l’Université Laval

ISSN

0034-379X (imprimé)

1703-8138 (numérique)

Découvrir la revue

Citer cet article

Cardin, J.-R. (1962). L’Analyse des conventions collectives de travail :

commentaires. Relations industrielles / Industrial Relations, 17(1), 10-14.

https://doi.org/10.7202/1037353ar

Tous droits réservés @ C Département des relations industrielles de l’Université Laval, 1962
Ce document est protégé par la loi sur le droit d'auteur. L'utilisation des services d'Érudit (y compris la reproduction) est assujettie à sa politique d'utilisation que vous pouvez consulter en ligne.

https://apropos.erudit.org/fr/usagers/politique-dutilisation/ 
négociateurs consultent nos études, il est possible qu'ils en retirent quelques éléments qu'ils voudront introduire dans leur propre contrat. Mais leur propre contrat, analysé comme les autres, sera peut-être utilisé par d'autres négociateurs. De toute façon, il est indéniable que les contrats collectifs s'influencent réciproquement. C'est là la preuve que le domaine des conventions collectives est un domaine vraiment dynamique; c'est pourquoi l'analyse des contrats collectifs est rarement facile et rarement routinière.

\section{Commentaires}

\section{Jean-Réal Cordin}

La convention collective, quoi qu'on en ait dit ou qu'on en dise encore aujourd'hui, demeure le pivot des relations industrielles en Amérique du Nord, l'instrument par excellence à l'aide duquel, dans les secteurs syndiqués de l'économie, les conditions de travail et d'emploi sont définies, le procédé selon lequel la vie entière de l'entreprise s'organise et se poursuit au jour le jour.

M. Quinet a voulu nous faire voir quelques aspects méthodologiques d'analyse des conventions collectives telles qu'elles s'expriment au Canada présentement. Ce faisant, il rejoint, avec le Ministère canadien du Travail, la préoccupation nécessaire que l'on retrouve chez d'autres organismes spécialisés dans les questions du travail, comme le Bureau international du Travail ou encore le Département du Travail des Etats-Unis qui a publié des études portant sur lanalyse des principales clauses de conventions collectives en vigueur chez nos voisins du Sud, ou en d'autres pays industrialisés.

L'analyse et l'appréciation des conventions collectives de travail prend d'autant plus d'importance dans le contexte nord-américain, que presque tous les aspects du statut des parties en présence, des droits respectifs de chacune d'elles, de leurs devoirs réciproques, des conditions de travail et d'emploi des travailleurs-individus, du règlement des conflits susceptibles de survenir dans l'administration des relations industrielles, ainsi qu'une large part de la sécurité sociale et du bien-être des salariés de l'industrie sont, en Amérique du Nord, donc au Canada, réglés au moyen de la convention de travail, de caractère privé et contractuel. 
C'est donc par l'étude des termes des conventions en vigueur à un moment donné, dans les différents secteurs industriels, que l'on peut déceler la condition économique, le statut social, le degré de protection et de sécurité, au lieu du travail et dans l'ensemble de l'économie, des travailleurs salariés de l'industrie nord-américaine. Cette situation offre un contraste assez marqué avec ce qui existe chez certains pays européens, par exemple, ou la majeure partie, sinon la totalité de ces questions sont résolues par voie législative et réglementaire, et où la convention collective elle-même revêt une allure statutaire et acquiert, dans un tel contexte juridique, valeur de règlement pré-établi qui s'impose aux parties sur le plan privé. La convention collective étant donc ce qu'elle est chez-nous, tend à constituer la seule source d'information sur les ingrédients composant nos relations industrielles, lesquels nous avons énumérés brièvement plus haut. Le travail de $M$. Quinet s'inscrit sans aucun doute dans la série des travaux nécessaires en cette matière.

En ce qui concerne la préparation du code d'analyse des conventions collectives de travail en vigueur, serait-il souhaitable de classifier les conventions, non seulement selon les catégories industrielles du Bureau fédéral de la Statistique, et selon les provinces, mais aussi en tenant compte, autant que possible, du type structurel des syndicats impliqués? J'entends par là, les différents types syndicaux afférents au mode de leur recrutement, au caractère professionnel ou industriel de leurs membres: syndicats dhommes de métier hautement qualifiés; syndicats groupant plusieurs degrés de compétence à l'intérieur de leurs cadres; syndicats dont les effectifs présentent un caractère homogène quant aux qualifications et aux fonctions de leurs membres; syndicats industriels, i.e., ne tenant pas compte des démarquations de métiers ou de compétence, mais englobant sur la base de tout un secteur, ou même de plusieurs secteurs industriels, les salariés de ces secteurs quels quils soient.

J'avoue qu'il peut ètre assez difficile souvent, de retrouver dans le menu ces différents types, mais une certaine classification pourrait sûrement être faite. Je dis ceci car on sait combien, en pratique, certaines clauses de conventions sont influencées dans leur contenu et leur importance par le type du syndicat négociateur. Ce regroupement des syndicats négociateurs, selon certains types structurels pré-établis, pourrait sûrement éclairer le pourquoi et le comment de certaines clauses, de leurs variations, de leur fréquence en certains secteurs industriels, 
etc. Et ceci, même si une classification par industrie donne déjà souvent une indication sur le type de syndicat concerné.

Dans la collection et la classification des conventions, la date de signature de ces conventions sert à les classer par année, ou par période de temps. Ce point, à mon sens, est important pour la constitution de séries historiques afin de constater, par exemple, l'évolution d'une clause en particulier dans une même industrie, ou son entrée ou sa disparition dans différents secteurs industriels. Il est aussi important pour permettre de tenir à jour le répertoire des clauses en vigueur en éliminant du code d'analyse les clauses tombées en désuétude.

Je crois aussi que non seulement quelques-unes parmi les clauses de conventions collectives nécessitent une définition que l'auteur appelle sabstraite " ou en tout cas dépouillée de toute contingence de formulation, de modalités mais que toutes les clauses ont besoin d'une définition abstraite afin que l'on puisse réellement catégoriser toutes les formulations qui peuvent exister d'un même type de clause et parvenir ainsi à l'établissement de secteurs aptes à couvrir la diversité de toutes les clauses ou la totalité des formulations qui se rencontrent dans les conventions étudiées.

A un moment de son analyse, lauteur nous indique que des choix sont à faire pour reconnaitre la substance mème d'une clause, ou d'un item de la convention, car la même terminologie peut vouloir signifier des réalités différentes quant à leur contenu. Après avoir choisi ce qu'une expression devra signifier pour qu'elle puisse être classifiée dans un des secteurs du code en usage, on peut se demander ce qu'il advient des clauses dont la terminologie est identique à celle du code, mais dont le contenu ne convient pas au secteur? Sont-elles simplement abandonnées ou reclassifiées selon le secteur où leur contenu des destine?

Il arrive souvent que les clauses de différentes conventions collectives ont une formulation identique et semblent, de ce fait, avoir la même portée et prêter à des conséquences semblables d'une convention à l'autre. Cependant, si l'on considère l'économie générale de chaque convention, et si l'on tient en mémoire les règles juridiques d'interprétation des contrats, qui à n'en pas douter, s'appliquent aux contrats collectifs de travail, il arrive qu'on ne peut souvent établir la portée réelle d'une clause qu'en l'étudiant en relation avec d'autres clauses qui viennent en limiter l'application, en altérer les effets, ou en préciser le sens. Ce qui veut dire que des clauses formulées d'une façon iden- 
tique, ne comportent pas nécessairement les mêmes conséquences pour les parties contractantes, ne signifient pas les mêmes obligations et ne peuvent pas être, par conséquent, traitées de la même façon. Nous pouvons rencontrer, par exemple, deux clauses traitant des droits de la direction au début de la convention, mais leur signification pourra varier considérablement selon les limitations qui y seront apportées par la clause traitant des griefs, et de leur procédure. Aussi: deux clauses traitant de l'application de l'ancienneté en cas de mises à pieds, bien que formulées d'une façon semblable, pourront recevoir une application différente selon la définition de l'expression «mise à pied 》 ou licenciement, ou encore selon les distinctions qui seront faites de ces expressions ailleurs, dans les conventions. Comment le code d'analyse peut-il tenir compte de ces situations?

Le code tient-il compte des articles sur les droits de la direction, leur étendue, leurs limites, leur libellé, etc.? S'il ne le fait pas, étant donné limportance considérable et la signafication que ces articles revêtent maintenant (aussi bien par leur absence, que par leur présence d'ailleurs dans le contrat), c'est là une lacune qu'il y aurait sans doute lieu de combler.

La confection judicieuse et Putilisation d'un code d'analyse des conventions collectives en vigueur est un instrument de première nécessité pour la recherche en convention collective et en relations industrielles plus généralement. Je ne soulignerai ici que deux exemples de problèmes qui appellent la recherche en convention collective à l'heure présente: ce sont l'automation et l'extension des cadres syndicaux à de nouveaux secteurs de salariés (professionnels, techniciens, etc.). La convention collective, à cause de phénomènes de cette sorte en relations industrielles, prend des dimensions nouvelles, acquiert une signification accrue pour les parties en présence, force chez elles la réflexion sur les processus d'accommodement possibles, et les formules de compromis utilisables.

Il est urgent, à mesure que le progrès technique, l'automation, tend à révolutionner les méthodes de production, que l'on puisse voir à quel point ces développements affectent le régime de la convention collective. Comment les syndicats des secteurs automatisés ou touchés par le progrès technologique, par exemple, entendent-ils contrôler, réglementer l'adoption de nouvelles méthodes de production, ou l'entrée de machinerie nouvelle? Comment essaient-ils, à tout le moins, d'atténuer les effets de ces changements sur les conditions de travail de leurs mem- 
bres, sur le rythme de leur travail, sur les standards établis de production, sur le contenu des tâches, etc.

Ces développements sont causes de nouvelles exigences syndicales qui se reflètent non seulement dans l'introduction de nouveaux articles dans les contrats collectifs, tendant à prévoir, à contrôler ou empêcher l'introduction de nouveaux procédés ou de nouvelles machines, mais encore dans la révision des clauses déjà existantes et qui sont directement affectées par ces changements: ancienneté, plan de pension, indemnités de licenciement, sécurité syndicale, âge de la retraite, etc.

L'analyse des conventions collectives est essentielle afin, non seulement de fournir un répertoire de clauses nouvelles ou modifiées, mais surtout pour permettre à l'aide de comparaisons dans le temps et selon les secteurs industriels, une connaissance détaillée de tels problèmes et de leur soludtion au moyen de la convention collective. Une telle analyse sert à orienter la recherche au fur et à mesure que de nouveaux secteurs la réclament avec une intensité accrue.

XVIle Congrès des Relations industrielles

Québec les 30 avril et ler mai 1962

\section{LA FONCTION DE CONSEIL DANS LE PROCESSUS DE DECISION}

Cette année le 17e Congrès des Relations industrielles, organisé par le Département des relations industrielles de la Faculté des sciences sociales, aura lieu au Château Frontenac, les 30 avril et ler mai.

Les participants étudieront la fonction de conseil, so nature, so raison d'être et son rôle dans l'entreprise, le syndicat et les organismes publics.

Tous sont cordialement invités à s'inscrire. 